Article

\title{
Some Connections between Classical and Nonclassical Symmetries of a Partial Differential Equation and Their Applications
}

\author{
Chaolu Temuer ${ }^{1, *}$, Laga Tong ${ }^{2}$ and George Bluman ${ }^{3}$ \\ 1 Arts and Sciences College of Shanghai Maritime University, Shanghai 201306, China \\ 2 Mathematics Department of Inner Mongolia University of Technology, Huhhot 010051, China; \\ lagalaga@163.com \\ 2 Mathematics Department of University of British Columbia, Vancouver, BC V6T 1Z2, Canada; \\ bluman@math.ubc.ca \\ * Correspondence: tmchaolu@shmtu.edu.cn
}

Received: 2 March 2020; Accepted: 24 March 2020; Published: 3 April 2020

\begin{abstract}
Essential connections between the classical symmetry and nonclassical symmetry of a partial differential equations (PDEs) are established. Through these connections, the sufficient conditions for the nonclassical symmetry of PDEs can be derived directly from the inconsistent conditions of the system determining equations of the classical symmetry of the PDE. Based on the connections, a new algorithm for determining the nonclassical symmetry of a PDEs is proposed. The algorithm make the determination of the nonclassical symmetry easier by adding compatibility extra equations obtained from system of determining equations of the classical symmetry to the system of determining equations of the nonclassical symmetry of the PDE. The findings of this study not only give an alternative method to determine the nonclassical symmetry of a PDE, but also can help for better understanding of the essential connections between classical and nonclassical symmetries of a PDE. Concurrently, the results obtained here enhance the efficiency of the existing algorithms for determining the nonclassical symmetry of a PDE. As applications of the given algorithm, a nonclassical symmetry classification of a class of generalized Burgers equations and the nonclassical symmetries of a KdV-type equations are given within a relatively easier way and some new nonclassical symmetries have been found for the Burgers equations.
\end{abstract}

Keywords: classical and nonclassical symmetries; connection; partial differential equation

\section{Introduction}

The nonclassical symmetry method, proposed by G. Bluman et al. in [1], is one of generalization of the classical Lie symmetry method for obtaining exact solutions of nonlinear PDEs which can not be obtained through its classical symmetries [2,3]. It has a close relation with the 'direct' method, Bäcklund transformations and functionally invariant solutions etc [4-6].

Determining classical and nonclassical symmetries of a PDE is equivalent to exactly solving the so-called the systems of determining equations arising from the invariance of the PDE under such symmetry transformations. There exist algorithms and software packages [7] to produce such systems of determining equations. However, to obtain nonclassical symmetries, exactly solving the system is a hard task due to its nonlinearity, unlike the situation for classical symmetries [8]. For this reason, nonclassical symmetries of many PDEs in physics and mechanics have not been found [9]. In [10-14], the authors investigate ways of simplifying the solution of the system of nonlinear determining equations based on the Gröebner basis method and Wu's method. In both methods, a system of determining equations is regarded as one of differential polynomials. Therefore, the set 
of solutions (equivalent to the set of symmetries of the PDE) of the system of determining equations is the same as the set of zero points (algebra variety) of the corresponding system of differential polynomials. Consequently, the problem of solving the system of determining equations is turned to determining the set of zero points of the corresponding polynomial system. This is suitably dealt with within the frameworks of the Gröebner basis method and Wu's method. These methods lead to the development of algorithms for directly dealing with the system of determining equations. However, in these algorithms, the computation of the differential Gröebner basis or the characteristic set of the system of corresponding determining equations is a difficult task and this is hardly implemented for higher dimensional PDEs [8]. Recently, in [15], we investigate an alternative algorithm to construct nonclassical symmetries of a PDE basing on so called 'potential conditions' and Wu's method. The conditions come from an 'observation' of link between classical and nonclassical symmetries of the PDE. However, the algorithm still depends on the calculation of the characteristic set of the determining system.

The aim of this article was to further investigate the more efficient way for determining nonclassical symmetry of a PDE. Particularly, some essential connections between the classical and nonclassical symmetries of a PDE are established through comparing the determining polynomials of the nonclassical symmetries with those of the classical symmetries. By the connections, some additional information on the existence of nonclassical symmetries of a PDE can be derived directly from the classical symmetries of the PDE. In particular, the connections provide supplementary conditions for nonclassical symmetries so that the corresponding nonlinear determining system is easier to solve. This leads to a more efficient method to determine the nonclassical symmetry of a PDE without calculating such characteristic set of the determining system and having these 'observations'. Our results not only reveal the more close connection between classical and nonclassical symmetries of a PDE, but also improve the efficiency of the methods in [10-15].

The rest of this paper is organized as follows. In Section 2, we give some preliminaries on the classical and nonclassical symmetries of a PDE and present a reduction formula of differential polynomial with respect to a differential ascending chain. In Section 3, we present our main results on connection between classical and nonclassical symmetries of a PDE in terms of the determining polynomial systems of the symmetries and inclusions connections of their zero point sets. In Section 4, as applications of the obtained connections, nontrivial nonclassical symmetries are found for both a class of generalized Burgers equations and system of $\mathrm{KdV}$-type equations in a relatively easier way. In Section 5, we summarize our results and include some concluding remarks.

\section{Preliminary}

\subsection{Notations}

Let $\mathbf{x}=\left(x_{1}, x_{2}, \ldots, x_{p}\right)$ and $\zeta=\left\{\xi_{1}, \xi_{2}, \ldots, \xi_{q}\right\}$ be independent and dependent variables, respectively. Let $\mathbb{N}_{0}$ be the set of non-negative integers. For a multi-index $\alpha=\left\{\alpha_{1}, \ldots, \alpha_{p}\right\} \in \mathbb{N}_{0}^{p}$, we denote the differential operator $\partial^{|\alpha|} / \partial_{x_{1}}^{\alpha_{1}} \partial_{x_{2}}^{\alpha_{2}} \ldots \partial_{x_{p}}^{\alpha_{p}}$ as $D^{\alpha}$ with order $|\alpha|=\sum_{i=1}^{p} \alpha_{i}$; we use $\partial^{k} \zeta$ to denote the set $\left\{D^{\alpha} \xi_{i}:|\alpha| \leq k, i=1,2, \ldots, q\right\}$ for a fixed $k \in \mathbb{N}_{0}$. Let $\mathcal{K}_{\mathbf{x}}$ be a characteristic zero field of differential functions of $\mathbf{x}$ and let $\mathcal{K}_{\mathbf{x}}[\zeta]$ be the differential polynomial ring in indeterminates $\partial^{k} \zeta$ for all non-negative integer $k$ over $\mathcal{K}_{\mathbf{x}}$.

A system of differential equations $\left\{p_{1}=0, p_{2}=0, \ldots, p_{s}=0\right\}$ where $p_{i}$ are polynomials in $\mathcal{K}_{\mathbf{x}}[\zeta]$ is denoted shortly as $\mathcal{D}=0$ where $\mathcal{D}$ is the set of left side polynomials of the equations, i.e., $\mathcal{D}=\left\{p_{1}, p_{2}, \ldots, p_{s}\right\} \subset \mathcal{K}_{\mathbf{x}}[\zeta]$. For a system of differential equations $\mathcal{D}=0$ in polynomial form, one has a corresponding system of differential polynomials $\mathcal{D}$. Use $Z(\mathcal{D})$ to denote the set of zero points of the differential polynomial system $\mathcal{D}$ over field $\mathcal{K}_{\mathbf{x}}$. Thus the solution set to the system of equations $\mathcal{D}=0$ is the same as the $\operatorname{set} Z(\mathcal{D})$.

Under a differential polynomial rank (order), the coefficient of the leading derivative of a differential polynomial is called initial of the polynomial. The partial derivative of a differential 
polynomial with respect to its leading derivative is called the separant of the polynomial. For a differential polynomial system $\mathcal{D}$, a product of initials and separants of the polynomials in the system is called IS product of the system, denote it as IS or IS $(\mathcal{D})$.

For two systems $\mathcal{D}$ and $Q$ of differential polynomials, we denote $Z(\mathcal{D} / Q)=Z(\mathcal{D}) \backslash Z(Q)$. in $\mathrm{Z}(\mathcal{D})$.

The notation $\left.f\right|_{Z(\mathcal{D})}$ denotes the set of values of the differential polynomial $f \in \mathcal{K}_{\mathbf{x}}[\zeta]$ at all points

In all examples, we use the graded lexicographic differential polynomial rank $[10,12,16,17]$ for differential polynomials in $\mathcal{K}_{\mathbf{x}}[\zeta]$ with no further elaboration.

\subsection{A Reduction Formula}

A reduction of a differential polynomial with respect to another one or an ascending chain of differential polynomials is a basic operation in polynomial algebra. A reduction formula frequently used is the pseudo-reduction given in $[16,17]$. It has been used in the methods given in $[7,10]$ and $[18,19]$ under different names such as Kolchin-Ritt reduction and Ritt-Wu reduction. We apply the reduction in this article for setting up a connection between classical and nonclassical symmetries of a PDE and analyze the set of zero points of a system of differential polynomials.

The reduction is related to the concept of the differential ascending chain of a differential polynomial system given below.

Definition 1. A differential ascending chain is a finite set of differential polynomials

$$
\mathcal{C S}=\left\{\mathcal{F}_{1}, \mathcal{F}_{2}, \ldots, \mathcal{F}_{s}\right\} \subset \mathcal{K}_{\mathbf{x}}[\zeta],
$$

if, under a differential polynomial rank $\prec$, it has the two properties showing below:

(a) $\mathcal{F}_{1} \prec \mathcal{F}_{2} \prec \ldots \prec \mathcal{F}_{s}$;

(b) $\mathcal{F}_{j}$ is reduced with respect to $\mathcal{F}_{i}$ (the terms in $\mathcal{F}_{j}$ can not be eliminated by $\mathcal{F}_{i}$ ) for $i=1,2, \ldots, j-1$.

The Ritt-Wu reduction for a differential polynomial $f \in \mathcal{K}_{\mathbf{x}}[\zeta]$ with respect to a differential ascending chain $\mathcal{C S} \subset \mathcal{K}_{\mathbf{x}}[\zeta]$ is given as follows.

Theorem 1. For a differential polynomial $h \in \mathcal{K}_{\mathbf{x}}[\zeta]$ and a differential ascending chain $\mathcal{C S} \subset \mathcal{K}_{\mathbf{x}}[\zeta]$, there exist differential operators $\mathfrak{D}_{i}=\sum_{\beta \in \mathbb{N}_{0}^{p}} Q_{i}^{\beta} D_{i}^{\beta}$ with $Q_{i}^{\beta} \in \mathcal{K}_{\mathbf{x}}[\zeta]$ and an IS product IS of $\mathcal{C} \mathcal{S}$ such that

$$
\mathrm{IS} \cdot h=\sum_{\mathcal{F}_{i} \in \mathcal{C} \mathcal{S}} \mathfrak{D}_{i} \mathcal{F}_{i}+R,
$$

where the $R \in \mathcal{K}_{\mathbf{x}}[\zeta]$, called the remainder of $h$ with respect to $\mathcal{C S}$ and denoted by $R=\operatorname{Rem}(h / \mathcal{C S})$, is reduced with respect to $\mathcal{C S}$ (any term in $R$ cannot be eliminated further by the polynomials in $\mathcal{C S}$ ) and the $D_{i}^{\beta}$ is the total differential operator related to $\mathcal{F}_{i} \in \mathcal{C S}$ with respect to $\beta \in \mathbb{N}_{0}^{p}$.

The Equation (2) is proved by successively removing from $h$ all terms that are differentiation of the leading derivatives of the elements of $\mathcal{C S}$ under a polynomial order until the procedure cannot be continued (the details are seen in [16-18]). The procedure of building Equation (2) is constructive and can be implemented by a computer algebra system [12]. For a differential polynomial system $\mathcal{D}$, we use the notation

$$
\operatorname{Rem}(\mathcal{D} / \mathcal{C S})=\{\operatorname{Rem}(h / \mathcal{C S}) \text { for } h \in \mathcal{D}\}
$$

In addition, a basic algorithm used in this article is the well-known Wu's algorithm (also called the characteristic set algorithm) for constructing a differential characteristic set for any given finite differential polynomial system. We use the algorithm to reduce a system of determining equations 
of the symmetries of a PDE into a differential ascending chain. The details of the algorithm appear in [18-21].

\subsection{Classical and Nonclassical Symmetries of a PDE}

For simplicity, we consider the case of a scale PDE. Actually, the results presented in the article hold in the case of a system of PDEs.

A more detailed discussion on the nonclassical symmetry for a general PDEs is given in [10].

Let an $k$ th order PDE

$$
\mathrm{F}[u]=\mathrm{F}\left(\mathbf{x}, \partial^{k} u\right)=0,
$$

be given with independent variables $\mathbf{x}$ and one dependent variable $u$. Suppose $\mathrm{F}$ is a polynomial in its arguments.

Let

$$
\mathcal{X}^{\prime}=\sum_{i=1}^{p} \xi_{i}^{\prime} \partial_{x_{i}}+\eta^{\prime} \partial_{u}
$$

be the generator of classical symmetry of Equation (3) where $\xi_{i}^{\prime}=\xi_{i}^{\prime}(\mathbf{z})$ and $\eta^{\prime}=\eta^{\prime}(\mathbf{z})$ are the infinitesimal functions of the generator with independent variables $\mathbf{z}=\left(x_{1}, x_{2}, \ldots, x_{p}, u\right)$.

The system of determining equations of generator Equation (4) satisfied by $\xi_{i}^{\prime}(\mathbf{z})$ and $\eta^{\prime}(\mathbf{z})$ is derived from the invariance criterion: $\operatorname{Pr} \mathcal{X}^{\prime}(\mathrm{F})=0$ when $\mathrm{F}=0$. Here $\operatorname{Pr} \mathcal{X}^{\prime}$ is the prolongation of $\mathcal{X}^{\prime}$ on the jet space of $u$. Explicitly solving the system, one determines generator Equation (4), i.e., the classical symmetry of PDE Equation (3).

The generator of the nonclassical symmetry of Equation (3)

$$
\mathcal{X}=\sum_{i=1}^{p} \xi_{i} \partial_{x_{i}}+\eta \partial_{u}
$$

is obtained by requiring that both Equation (3) and its invariance surface equation

$$
\psi=\sum_{i=1}^{p} \xi_{i} u_{x_{i}}-\eta=0,
$$

are simultaneously invariant under the symmetry transformation of Equation (5). The invariance criterion: $\operatorname{Pr} \mathcal{X}(\mathrm{F})=0$ when $F=0$ and $\psi=0$ leads to the system of determining equations for nonclassical symmetry Equation (5). The system is a nonlinear PDEs for the infinitesimal functions $\xi_{i}=\xi_{i}(\mathbf{z})$ and $\eta=\eta(\mathbf{z})$ that appear in Equations (5) and (6). Thus, in principle, after exactly solving the system, one finds the nonclassical symmetry Equation (5). However, this is, in general, a hard task $[8,10]$.

The concept of equivalent generators plays a key rule in this article.

Definition 2. Two first order differential operators $\mathcal{X}^{\prime}$ and $\mathcal{X}$ of the form as Equations (4) and (5) are called equivalent if they differ by a nonzero differential function multiplier $\lambda(\boldsymbol{z})$, i.e., $\mathcal{X}^{\prime}=\lambda(\boldsymbol{z}) \mathcal{X}$.

Equivalent symmetry generators of a PDE generate the same reduced solutions of the PDE.

Remark 1. In this article, without loss of generality, we assume $\xi_{1} \neq 0$ in Equation (5). Otherwise, we successively assume $\xi_{k} \neq 0$ and $\xi_{i}=0, i=1,2, \cdots, k-1$ and discuss each case separately. Hence, by Definition 2 , we set $\xi_{1}=1$. Conventionally, in the case of an evolution equation case, each $\xi_{1}$ is the coefficient of $\partial_{t}$ in generator $\mathcal{X}$ corresponding to the time variable, usually denoted by $\tau$. Hence, in the regular case of the nonclassical symmetries for an evaluation equation, we set $\tau=1$. 
In the following, let $\mathcal{D}^{\prime}=0$ and $\mathcal{D}=0$ be the systems of determining equations for the symmetry generators $\mathcal{X}^{\prime}$ and $\mathcal{X}$ of PDE Equation (3). The sets of the left hand sides $\mathcal{D}^{\prime} \subset \mathcal{K}_{\mathbf{z}}\left[\Lambda^{\prime}\right]$ and $\mathcal{D} \subset \mathcal{K}_{\mathbf{z}}[\Lambda]$ of the two systems are called determining polynomial systems of the classical and nonclassical symmetries respectively. Then, the zero point sets $Z\left(\mathcal{D}^{\prime}\right)$ and $Z(\mathcal{D})$ are the sets of the classical and nonclassical symmetries of the PDE.

In convenience, we use the following additional notations: $\Lambda^{\prime}=\left(\xi_{1}^{\prime}, \ldots, \xi_{p}^{\prime}, \eta^{\prime}\right), \Lambda=$ $\left(\xi_{2}, \ldots, \xi_{p}, \eta\right), \partial_{\mathbf{z}}=\left(\partial_{x_{1}}, \partial_{x_{2}}, \ldots, \partial_{x_{p}}, \partial_{u}\right)$ and $\tilde{\partial}_{\mathbf{z}}=\left(\partial_{x_{2}}, \partial_{x_{3}}, \ldots, \partial_{x_{p}}, \partial_{u}\right)$. Thus, generators Equations (4) and (5) can be written shortly as $\mathcal{X}^{\prime}=\Lambda^{\prime} \cdot \partial_{\mathbf{z}}$ and $\mathcal{X}=\partial_{x_{1}}+\Lambda \cdot \tilde{\partial}_{\mathbf{z}}$ by scalar product rule of finite dimensional vector space.

Our main goal is to determine some connections between the systems $\mathcal{D}$ and $\mathcal{D}^{\prime}$.

\section{Connections between Classical and Nonclassical Symmetries of a PDE}

It is well-known that the set of classical symmetries of a PDE is a subset of the nonclassical symmetries of the PDE. Hence, the classical symmetry of a PDE has equivalent expressions in the coordinates $\Lambda^{\prime}$ and $\Lambda$.

Therefore, we use a map between any $\Lambda^{\prime}=\left(\xi_{1}^{\prime}, \cdots, \xi_{p}^{\prime}, \eta^{\prime}\right)$ with $\xi_{1}^{\prime} \neq 0$ and $\Lambda=\left(\xi_{2}, \cdots, \xi_{p}, \eta\right)$ as coordinately

$$
\xi_{i}^{\prime}=\xi_{1}^{\prime} \xi_{i}, \quad \eta^{\prime}=\xi_{1}^{\prime} \eta, \quad i=2, \ldots, p
$$

If a nonclassical symmetry $\mathcal{X}=\partial_{x_{1}}+\Lambda \cdot \tilde{\partial}_{\mathbf{z}}$ represents a classical symmetry, then there exists an equivalent $\mathcal{X}^{\prime}=\Lambda^{\prime} \cdot \partial_{\mathbf{z}}$ such that $\Lambda^{\prime}=\left(\xi_{1}^{\prime}, \cdots, \xi_{p}^{\prime}, \eta^{\prime}\right) \in Z\left(\mathcal{D}^{\prime}\right)$ and $(1, \Lambda)=\left(1, \xi_{2}, \cdots, \xi_{p}, \eta\right) \in Z(\mathcal{D})$ have a unified proportional relationship. Hence, the transformation Equation (7) map a classical symmetry expressed by an $\Lambda \in Z(\mathcal{D})$ into equivalence one expressed by the corresponding $\Lambda^{\prime} \in Z\left(\mathcal{D}^{\prime}\right)$ and vice versa.

Let $\prec$ be a differential polynomial rank on $\mathcal{K}_{\mathbf{z}}\left[\Lambda^{\prime}\right]$ with $\xi_{1}^{\prime}$ having the highest rank, i.e., $\xi_{j}^{\prime} \prec$ $\xi_{1}^{\prime}, j \neq 1$. Let $C^{\prime}$ be an ascending chain form of $\mathcal{D}^{\prime}$ which can be obtained by Wu's algorithm under the rank. In [12], we have proved that $Z\left(C^{\prime}\right)=Z\left(\mathcal{D}^{\prime}\right)$ for a PDE if $\operatorname{IS}\left(C^{\prime}\right) \neq 0$. Hence, the set $Z\left(C^{\prime}\right)$ represents the classical symmetry of the PDE under the conditions that the initials of $C^{\prime}$ are not zero.

Remark 2. Here, we emphasize that it is unnecessary for $C^{\prime}$ to be a characteristic set of $\mathcal{D}^{\prime}$, which is required in the algorithms given in $[12,15]$. Here, the cost of calculating $C^{\prime}$ is much less than that of calculating the characteristic set of $D^{\prime}$. In the latter, it is involved the calculations of integral conditions of a system which is a recognized hard problem [16-18].

In evidence, the set $C^{\prime}$ is an irreducible ascending chain due to its linearity.

Throughout this article, we assume that the initials of the differential polynomials in $C^{\prime}$ are not zero, i.e.,

$$
\operatorname{IS}\left(C^{\prime}\right) \not \equiv 0
$$

Remark 3. Assumption Equation (8) is not critical for a PDE. Since the the polynomials in $C^{\prime}$ are linear, the initials of $C^{\prime}$ are polynomials in terms of the independent variable $z$, so they are never zero if the PDE does not contain parameters.

Now, we obtain the connections between classical and nonclassical symmetries of a PDE, which provides sufficient conditions to determine the existence of a nontrivial nonclassical symmetry.

Theorem 2. For an $\Lambda \in Z(\mathcal{D})$, if, after using transformations Equation (7) for a $\xi_{1}^{\prime} \neq 0$, the $C^{\prime}$ as a differential polynomial system in $\mathcal{K}_{\mathbf{z}}\left[\Lambda, \xi_{1}^{\prime}\right]$ has no zero points, i.e., $\mathrm{Z}\left(C^{\prime}\right)=\varnothing$ (equivalently, equations $C^{\prime}=0$ is inconsistence), then the operator $\mathcal{X}=\partial_{x_{1}}+\Lambda \cdot \tilde{\partial}_{\mathbf{z}}$ is a nontrivial nonclassical symmetry of the PDE. 
Proof. If the $\mathcal{X}$ is equivalent to a classical symmetry, then there exists $\Lambda^{\prime}=\left(\xi_{1}^{\prime}, \cdots, \xi_{p}^{\prime}, \eta^{\prime}\right) \in Z\left(C^{\prime}\right)$ such that $\mathcal{X}^{\prime}=\Lambda^{\prime} \cdot \partial_{\mathbf{Z}}$ and the $\mathcal{X}$ are equivalent under transformation Equation (7). This means $\mathrm{Z}\left(\mathrm{C}^{\prime}\right) \neq \varnothing$. This is a contradiction with respect to the assumption of the theorem.

Use the notation $\mathcal{D}^{\prime \prime}$ to denote the set of the differential polynomials in $C^{\prime}$ whose leading derivatives are the derivatives of $\xi_{1}^{\prime}$ with respect to z. Thus, for any subset $Q \subset C^{\prime}$, one has the following corollaries of this theorem.

Corollary 1. The Theorem 2 is also true when replacing $C^{\prime}$ in the theorem with any subset $Q \subset C^{\prime}$. In particular, when $Q$ is $\mathcal{D}^{\prime \prime}$ or the complement set $C^{\prime} \backslash \mathcal{D}^{\prime \prime}$, the theorem is still true.

Let $Q \subset C^{\prime}$. Use $D Q$ to denote a set of polynomials obtained from $Q$ or total derivatives of some polynomials in $Q$.

Then we have the following second corollary of Theorem 2.

Corollary 2. The Theorem 2 is also true when replacing $C^{\prime}$ in the theorem with $D C^{\prime}$ or $D Q$ for any subset $Q \subset C^{\prime}$.

Proof. The theorem is a direct conclusion of Theorem 2 and the inclusion relation $Z(Q) \subset Z(D Q)$.

In the following, based on Equation (2) and transformation Equation (7), we turn $C^{\prime} \subset \mathcal{K}_{\mathbf{z}}\left[\Lambda^{\prime}\right]$ into a system of differential polynomials in $\mathcal{K}_{\mathbf{z}}\left[\Lambda, \xi_{1}^{\prime}\right]$ so that it connects the classical symmetries $Z\left(C^{\prime}\right)$ and nonclassical symmetries $\mathrm{Z}(\mathcal{D})$.

From the structure of an ascending chain, we know that the both set $\mathcal{D}^{\prime \prime}$ and the complement set $C^{\prime} \backslash \mathcal{D}^{\prime \prime}$ are differential ascending chain in $\mathcal{K}_{\mathbf{z}}\left[\Lambda^{\prime}\right]$. Using transformation Equation (7), we change $C^{\prime}$ and $\mathcal{D}^{\prime \prime}$ to the systems in $\mathcal{K}_{z}\left[\Lambda, \xi_{1}^{\prime}\right]$ (again denote therm as $C^{\prime}$ and $\mathcal{D}^{\prime \prime}$ ). It is noticed that the rank $\prec$ on $\mathcal{K}_{\mathbf{z}}\left[\Lambda^{\prime}\right]$ induces a rank on $\mathcal{K}_{\mathbf{z}}\left[\Lambda, \xi_{1}^{\prime}\right]$ through $\xi_{i}^{\prime}$ matching $\xi_{i}$ and $\eta^{\prime}$ matching $\eta(i=2, \cdots, p)$. Obviously, it keeps the highest rank of $\xi_{1}^{\prime}$. Under the induced rank, we do the reduction

$$
C=\operatorname{Rem}\left(\left(C^{\prime} \backslash \mathcal{D}^{\prime \prime}\right) / \mathcal{D}^{\prime \prime}\right) \subset \mathcal{K}_{\mathbf{z}}\left[\Lambda, \xi_{1}^{\prime}\right],
$$

through Equation (2). In the reduction of $C$, the operation on $(\xi, \eta)$ is not involved. Therefore, due to the highest rank of $\xi_{1}^{\prime}$ and the linearity of both $C^{\prime}$ and transformations Equation (7), each element in $C$, as a differential polynomial in $\mathcal{K}_{\mathbf{z}}\left[\Lambda, \mathcal{\xi}_{1}^{\prime}\right]$ has the same rank and initials as its corresponding one in the $C^{\prime} \backslash \mathcal{D}^{\prime \prime} \subset \mathcal{K}_{\mathbf{z}}\left[\Lambda^{\prime}\right]$. Hence, the $C$ inherits the differential ascending chain property of the $C^{\prime} \backslash \mathcal{D}^{\prime \prime}$ as well as the linearity of its polynomials in their leading derivatives with Equation (8).

So far, we have turned $C^{\prime} \subset \mathcal{K}_{\mathbf{z}}\left[\Lambda^{\prime}\right]$ into the $C \subset \mathcal{K}_{\mathbf{z}}\left[\Lambda, \xi_{1}^{\prime}\right]$.

For the convenience, denote

$$
\mathcal{D}=\left\{h_{1}, h_{2}, \ldots, h_{n}\right\} \subset \mathcal{K}_{\mathbf{z}}[\Lambda],
$$

and

$$
C=\left\{r_{1}, r_{2}, \ldots, r_{m}\right\} \subset \mathcal{K}_{\mathbf{z}}\left[\Lambda, \xi_{1}^{\prime}\right]
$$

Thus, we have connection between $C$ and $C^{\prime}$ as follows.

Theorem 3. The set $Z(C)$ contains all classical symmetries $\mathcal{X}^{\prime}=\Lambda^{\prime} \cdot \partial_{\mathbf{z}}$ with $\Lambda^{\prime}=\left(\xi_{1}^{\prime}, \cdots, \xi_{p}^{\prime}, \eta^{\prime}\right)$ and $\xi_{1}^{\prime} \neq 0$ of PDE Equation (3) through transformation Equation (7). More precisely, $\mathrm{Z}\left(C^{\prime}\right) \subset \mathrm{Z}(C)$ holds by means of the equivalence in Definition 2.

Proof. For an $\Lambda^{\prime} \in Z\left(C^{\prime}\right)$, since a classical symmetry $\mathcal{X}^{\prime}=\Lambda^{\prime} \cdot \partial_{\mathbf{z}}$ with $\xi_{1}^{\prime} \neq 0$ is equivalent to a nonclassical symmetry of the same PDE, there is a $\Lambda=\left(\xi_{2}, \cdots, \xi_{p}, \eta\right) \in \mathrm{Z}(D)$ connecting $\mathcal{X}^{\prime}$ through 
transformations Equation (7). The construction procedure for $C$ immediately shows that $\Lambda \in Z(C)$. This ends the proof of the Theorem.

Now, we can establish a connection between classical and nonclassical symmetries of a PDE in terms of the dependence of the determining polynomials in $\mathcal{D}$ on the ones in $C$ as stated in the following theorem.

Theorem 4. For each $h \in \mathcal{D}$, there exist differential operators $\mathfrak{D}_{v}^{h}$ with coefficients in $\mathcal{K}_{\mathbf{z}}[\Lambda]$ and an IS product $\mathrm{IS}_{h}$ of $C$ such that the identities

$$
\mathrm{IS}_{h} \cdot h=\sum_{v=1}^{m} \mathfrak{D}_{v}^{h} r_{v}
$$

hold for all differential functions $\xi$ and $\eta$.

Proof. From the construction of $C$, for each $q^{\prime} \in C^{\prime} \backslash D^{\prime \prime}$, there exist some differential operators $\mathfrak{D}_{v}^{\prime}$ and an IS product IS' of $D^{\prime \prime}$ such that

$$
\mathrm{IS}^{\prime} \cdot q^{\prime}=\sum_{d_{v} \in \mathcal{D}^{\prime \prime}} \mathfrak{D}_{v}^{\prime} d_{v}+r
$$

from reduction Equation (2). Here the remainder $r=\operatorname{Rem}\left(q^{\prime} / \mathcal{D}^{\prime \prime}\right) \in C$ is reduced with respect to $D^{\prime \prime}$. The obtained identity with assumption Equation (8) implies

$$
\mathrm{Z}(C) \cap \mathrm{Z}\left(\mathcal{D}^{\prime \prime}\right) \subset \mathrm{Z}\left(C^{\prime}\right) \subset \mathrm{Z}(C) .
$$

Noticing that $D^{\prime \prime} \subset C^{\prime}$ and using above inclusions, we can easily prove that

$$
\mathrm{Z}\left(C \cup \mathcal{D}^{\prime \prime}\right)=\mathrm{Z}\left(C^{\prime}\right)
$$

Furthermore, the equality (11) shows that the differential ascending chain $C \cup D^{\prime \prime}$ is irreducible since the $C^{\prime}$ is irreducible.

On the other hand, for each $h \in \mathcal{D}$, there are some differential operators $\mathfrak{D}_{v}$ and an $I S_{h}$ product IS of $C$, such that

$$
\mathrm{IS}_{h} \cdot h=\sum_{r_{v} \in C} \mathfrak{D}_{v} r_{v}+R,
$$

from reduction Equation (2). Here the remainder $R=\operatorname{Rem}(h / C)$ is reduced with respect to $C$. It, from Equation (9), is also noticed that the $R$ is also reduced with respect to $D^{\prime \prime}$. Hence the remainder $R$ is reduced with respect to irreducible ascending chain $C \cup D^{\prime \prime}$.

Since $Z\left(C^{\prime}\right)=Z\left(D^{\prime}\right) \subset Z(D)$, the identity Equation (12) and Theorem 3 imply that the remainder $R=\operatorname{Rem}(h / C)$ is identically equal to zero on $Z\left(C^{\prime}\right)$, i.e.,

$$
\left.R\right|_{\mathrm{Z}\left(C^{\prime}\right)}=0 .
$$

Furthermore, the equality (11) indicates that

$$
\left.R\right|_{\mathrm{Z}\left(C \cup \mathcal{D}^{\prime \prime}\right)}=0,
$$

which yields $R \equiv 0$ in Equation (12) due to $r$ being reduced with respect to $C \cup \mathcal{D}^{\prime \prime}$ and the irreducibility of $C \cup \mathcal{D}^{\prime \prime}$. Consequently, the identities Equation (10) in the theorem are true.

Thus, the identities Equations (10) with (8) imply $\mathrm{Z}(C) \subset \mathrm{Z}(\mathcal{D})$. 
Summarizing the above discussions, we have the inclusions:

$$
\mathrm{Z}\left(C^{\prime}\right) \subset \mathrm{Z}(C) \subset \mathrm{Z}(\mathcal{D})
$$

Remark 4. Theorems 2-4 and Corollaries 1 and 2 give relationships between the classical and nonclassical symmetries of PDE Equation (3) in terms of the determining polynomial systems $C^{\prime}, C$ and $\mathcal{D}$ of the two kinds of symmetries, in which the set $C$ plays the rule of a bridge between classical and nonclassical symmetries of a PDE.

From the viewpoint of practical applications, the inclusions Equation (13) imply that more additional equations from $C$ can be used to extended system $\mathcal{D}$ for nonclassical symmetry. In fact, any subset $Q \subset C$ or $D Q$ is compatible with $\mathcal{D}$. Hence, we get some extended system of $\mathcal{D}$ by appending $Q$ or $D Q$ to $\mathcal{D}$. The extended system is more easily solved than the original $\mathcal{D}$ since it contains more equations. It may yield a nonclassical symmetry of the underling PDE.

Obviously, the above process of proving Equation (10) is constructive and forms an algorithm for constructing Equation (10).

The following is an illustrative example to show how well the above theorems and algorithm work well.

Example 1. We consider the Burgers - Huxley equation

$$
u_{t}=u_{x x}+u(u-1)(u-\sigma) .
$$

The whole classical symmetries admitted by the equation are obvious symmetries

$$
\mathcal{X}_{1}=\partial_{t}, \mathcal{X}_{2}=\partial_{x}
$$

corresponding to time and space translations for any $\sigma \in \mathbb{R}$.

We first establish identities Equation (10) following the procedure (algorithm) of proving Theorem 4.

Step 1. Computing $\mathcal{D}$ and $\mathcal{D}^{\prime}$. The systems of determining equations for a nonclassical symmetry $\mathcal{X}=\partial_{t}+\xi \partial_{x}+\eta \partial_{u}$ and a classical symmetry $\mathcal{X}^{\prime}=\tau^{\prime} \partial_{t}+\xi^{\prime} \partial_{x}+\eta^{\prime} \partial_{u}$ of the equation are given by $\mathcal{D}=\left\{h_{1}, h_{2}, h_{3}, h_{4}\right\}=0$ and $\mathcal{D}^{\prime}=0$ respectively, where

$$
\begin{aligned}
& h_{1}=\xi_{u u}, \\
& h_{2}=\eta_{u u}-2 \xi_{x u}+2 \xi_{u}, \\
& h_{3}=2 \eta_{x u}-\xi_{x x}+\xi_{t}+2 \xi_{x}-(3 u(u-1)(u-\sigma)+2 \eta) \xi_{u}, \\
& h_{4}=\eta_{x x}-\eta_{t}+2 \eta \xi_{x}+\left(\sigma-2 u-2 \sigma u+3 u^{2}\right) \eta+u(u-\sigma)(u-1)\left(2 \xi_{x}-\eta_{u}\right),
\end{aligned}
$$

and

$$
\begin{aligned}
& \mathcal{D}^{\prime}=\left\{\tau_{u}^{\prime}, \tau_{x}^{\prime}, \tau_{t}^{\prime}-2 \xi_{x}^{\prime}, \xi_{u}^{\prime}, \xi_{x x}^{\prime}-2 \eta_{x u}^{\prime}-\xi_{t}^{\prime}, \eta_{u u}^{\prime}\right. \\
& \left.\eta_{t}^{\prime}-\eta_{x x}^{\prime}+u(u-1)(u-\sigma)\left(2 \xi_{x}^{\prime}-\eta_{u}^{\prime}\right)+\left(\sigma+3 u^{2}-2(\sigma+1) u\right) \eta^{\prime}\right\}
\end{aligned}
$$

Step 2. Turning $\mathcal{D}^{\prime}$ into ascending chain form. Obviously, the system $\mathcal{D}^{\prime}$ is already an ascending chain form under rank $t \prec x \prec u \prec \xi^{\prime} \prec \eta^{\prime} \prec \tau^{\prime}$. Hence $C^{\prime}=\mathcal{D}^{\prime}$ and

$$
\mathcal{D}^{\prime \prime}=\left\{\tau_{t}^{\prime}-2 \tilde{\xi}_{x}^{\prime}, \tau_{x}^{\prime}, \tau_{u}^{\prime}\right\}
$$

with $\operatorname{IS}\left(C^{\prime}\right)=2 \not \equiv 0$. 
Step 3. Substituting the transformation Equation (7) with $\xi_{1}^{\prime}=\tau^{\prime}$ into $C^{\prime}$, then computing the reduction $\operatorname{Rem}\left(\left(C^{\prime} \backslash \mathcal{D}^{\prime \prime}\right) / \mathcal{D}^{\prime \prime}\right)$ and after deleting the common factor $\tau^{\prime} \neq 0$, we have the differential ascending chain $C=\left\{r_{1}, r_{2}, r_{3}, r_{4}\right\}$ under the induced rank $t \prec x \prec u \prec \xi \prec \eta$ of the rank in Step 2 . Here

$$
\begin{aligned}
& r_{1}=\xi_{u}, \\
& r_{2}=\eta_{u u} \\
& r_{3}=2 \eta_{x u}+\xi_{t}+2 \xi_{\xi x}-\xi_{x x}, \\
& r_{4}=\eta_{x x}-\eta_{t}+2 \eta \xi_{x}+\left(\sigma-2 u-2 \sigma u+3 u^{2}\right) \eta+u(u-\sigma)(u-1)\left(2 \xi_{x}-\eta_{u}\right),
\end{aligned}
$$

with $I S(C)=2$.

Step 4. Using reduction Equation (2), one obtains relations Equation (10) as follows

$$
\begin{aligned}
& h_{1}=D_{u} r_{1} \\
& h_{2}=r_{2}+2\left(\xi-D_{x}\right) r_{1}, \\
& h_{3}=r_{3}-(3 u(u-1)(u-\sigma)+2 \eta) r_{1}, \\
& h_{4}=r_{4} .
\end{aligned}
$$

This implies $Z(C) \subset Z(D)$, i.e., the right side inclusion of Equation (13) holds.

Obviously, the first classical symmetry in Equation (15) with $\xi_{1}^{\prime}=\tau \equiv 1$ is contained in $Z(C)$, i.e., Theorem 3 is satisfied. We notice that the $(\xi, \eta)$ with

$$
\begin{aligned}
& \xi(t, x, u)=a(x)=3 \tan \left(\frac{1}{2 \sqrt{2}}\left(x+24 c_{1}\right)\right) / 2 \sqrt{2}, \\
& \eta(t, x, u)=(1-2 u)\left(8 a(x)^{2}+9\right) / 48 .
\end{aligned}
$$

is in $Z(C)$ for $\sigma=1 / 2$ but does not correspond to any classical symmetry,i.e., $Z\left(C^{\prime}\right)=\varnothing$. This verified the Theorem 2. It also shows that in general $\mathrm{Z}(C)$ may be larger than the set of classical symmetries.

Rather, the $(\xi, \eta)$ with $\xi=(3 u-\sigma-1) / \sqrt{2}, \eta=-(3 / 2) u(u-1)(u-\sigma)$ which makes all $p_{i}=$ $0(i=1,2,3,4)$ in Equation (16), i.e., the $(\xi, \eta) \in Z(\mathcal{D})$. However, it is obvious that $q_{1} \neq 0$. Hence the $(\xi, \eta) \notin Z(C)$. This shows that $Z(C)$ is a proper subset of $Z(\mathcal{D})$ and the operator $\mathcal{X}=\partial_{t}+(3 u-\sigma-$ $1) / \sqrt{2} \partial_{x}-(3 / 2) u(u-1)(u-\sigma) \partial_{u}$ is a generator of a nontrivial nonclassical symmetry of Equation (14). Consequently, we properly have $\mathrm{Z}\left(C^{\prime}\right) \subset \mathrm{Z}(C) \subset \mathrm{Z}(\mathcal{D})$ in this example.

\section{Applications}

We give two examples to show the applications of Theorems 2-4 and Corollaries 1 and 2 to determine nontrivial nonclassical symmetries of a given PDE.

\subsection{A Nonclassical Symmetry Classification of a Class of Generalized Burgers Equations}

The nonclassical symmetry classification of a PDE with arbitrary functions is a hard problem in symmetry analysis of a PDE $[9,10]$. We solve this problem by using given Theorems 2-4 and Corollaries 1 and 2.

Consider the nonclassical symmetry classification of a class of generalized Burgers equations

$$
u_{t}+g(u) u_{x}-u_{x x}=f(u)
$$

with two arbitrary functions parameters $f(u)$ and $g(u)$, which covers a wide range of mathematical models describing various processes in physics, biology and ecology(see $[11,22-24]$ and references therein).

For the case $g(u)=0$, the equation represents: the Huxley equation if $f(u)=u^{2}(1-u)$; the Fisher's equation if $f(u)=u(1-u)$; the generalized Fisher's equations if $f(u)=u-u^{k}$ or 
$f(u)=u^{p}\left(1-u^{p-1}\right)$; the Chaffee - Infante or Newell - Whitehead equation if $f(u)=\beta u\left(1-u^{2}\right)$; the generalized KPP equation if $f(u)=\alpha u^{3}+\beta u^{2}+\rho u$; the Fitzhugh - Nagumo equation if $f(u)=u(1-u)(u-\alpha)$, etc;

For the case $g(u)=\alpha u$, the equation represents: the Burgers equation if $f(u)=0$; the Burgers Huxley equation if $f(u)=\beta u(1-u)(u-\gamma)$, etc;

For the case $g(u)=\alpha u^{c}(c \in R)$, the equation represents: the generalized Burgers - Fisher equation if $f(u)=\beta u\left(1-u^{c}\right)$; the generalized Burgers - Huxley equation if $f(u)=\beta u\left(1-u^{c}\right)\left(u^{c}-\gamma\right)$, etc.

In [22], the authors considered Equation (19) with $g(u)=\lambda u$ and gave the nonclassical (there Q-conditional) symmetry classification with respect to $f(u)$. For $g=0$, the complete nonclassical symmetry classification is given in [11]. In [23,24], the complete classical symmetry classification of the equations was given.

Now we consider the nonclassical symmetry classification of Equation (19) in general sense for two arbitrary parameters $f(u)$ and $g(u)$ by using the method given in the article.

The system of determining polynomials of classical symmetry $\mathcal{X}^{\prime}=\tau^{\prime} \partial_{t}+\xi^{\prime} \partial_{x}+\eta^{\prime} \partial_{u}$ of Equation (19) is given by

$$
\mathcal{D}^{\prime}=\left\{\begin{array}{l}
\tau_{u}^{\prime}, \tau_{x}^{\prime}, \tau_{t}^{\prime}-2 \xi_{x}^{\prime}, \xi_{u}^{\prime}, \eta_{u u}^{\prime} \\
\eta_{x x}^{\prime}+f^{\prime}(u) \eta^{\prime}+f(u)\left(2 \xi_{x}^{\prime}-\eta_{u}^{\prime}\right)-g(u) \eta_{x}^{\prime}-\eta_{t}^{\prime}, \\
2 \eta_{x u}^{\prime}+\xi_{x x}^{\prime}+g^{\prime}(u) \eta^{\prime}+g(u) \xi_{x}^{\prime}-\xi_{t}^{\prime} .
\end{array}\right.
$$

Under the order $t \prec x \prec u \prec \xi^{\prime} \prec \eta^{\prime} \prec \tau^{\prime}$, the system is already in differential chain form. Hence $C^{\prime}=\mathcal{D}^{\prime}$.

By using the given algorithm, we find the relationships Equation (10) between $\mathcal{D}$ and $C=$ $\left\{r_{1}, r_{2}, r_{3}, r_{4}\right\}$ as follows

$$
\begin{aligned}
& h_{1}=r_{3}+2(\xi-g(u)) r_{4}-2 D_{x} r_{4} \\
& h_{2}=r_{2}+(3 f(u)-2 \eta) r_{4} \\
& h_{3}=D_{u} r_{4} \\
& h_{4}=r_{1}
\end{aligned}
$$

where

$$
\begin{aligned}
& r_{1}=f(u) \eta_{u}+g(u) \eta_{x}-\eta_{x x}+\eta_{t}+2(\eta-f(u)) \xi_{x}-\eta f^{\prime}(u) \\
& r_{2}=2 \eta_{x u}-\xi_{x x}+(2 \xi-g(u)) \xi_{x}-\eta g^{\prime}(u)+\xi_{t} \\
& r_{3}=\eta_{u u} \\
& r_{4}=\xi_{u}
\end{aligned}
$$

with $\operatorname{IS}(C)=-2$.

From the transformation Equation (7) in this example, we have $C^{\prime} \ni \xi_{u}^{\prime}=\xi_{u} \tau^{\prime}$ which implies that Equations (19) admits a nonclassical symmetry when $r_{4} \neq 0$ and $Z(\mathcal{D}) \neq \varnothing$ from Theorem 2 . Therefore, based on the reasonable information we consider the cases of $r_{4} \neq 0$ and $r_{4}=0$ respectively.

Case 1: $r_{4} \neq 0$.

In the case, from Theorem 2 we can impose more restrictions on the system $\mathcal{D}=0$ so that it is solved easily and yields nontrivial nonclassical symmetries of the equation. It is observed that if one takes $\eta$ as $\eta_{u u} \neq 0$, then $\eta_{u u}^{\prime}=\eta_{u u} \tau^{\prime} \in C^{\prime}$ never be zero under transformation Equation (7) for $\tau^{\prime} \neq 0$. Hence, according to Theorem 2 , such $\xi$ and $\eta$ yield a nontrivial nonclassical symmetry of the equation. Based on the analysis, an ansatz of the infinitesimal functions that fits these conditions is $\xi=a(t, x) u+b(t, x)$ and $\eta=\alpha(t, x) u^{3}+\beta(t, x) u^{2}+\rho(t, x)+\gamma(t, x)$ with $\alpha^{2}(t, x)+\beta^{2}(t, x) \neq 0$ or $\alpha(t, x) \neq 0$. 
Remark 5. Actually, one takes $\eta=\sum_{i=0}^{n} \alpha_{i}(t, x) u^{i}$ in more general form. However, it is easy to deduce that $\alpha_{i} \equiv 0$ when $i \geq 4$.

Under these restrictions, system $\mathcal{D}=0$ is reduced significantly and solved easily. In particular, we have the following three groups of nonclassical symmetries.

$$
\xi=a u+b, \eta=\frac{1}{3}\left(c_{1}-a\right) u^{3}+a\left(c_{2}-b\right) u^{2}+\rho u+\gamma,
$$

for $f(u)=\frac{\left(2 a+c_{1}\right)}{9 a}\left(a\left(c_{1}-a\right) u^{3}+3 a\left(c_{2}-b\right) u^{2}+3 \rho u+3 \gamma\right), g(u)=c_{1} u+c_{2}$ where $a, b, c_{1}, c_{2}, \rho$ and $\gamma$ are arbitrary constants with $a \neq 0$ or $c_{2} \neq b$ or $c_{1} \neq a$;

$$
\xi=\frac{c_{1}}{4} u+b, \eta=\frac{1}{16} c_{1}^{2} u^{3}-\frac{1}{4} c_{1}\left(b-c_{2}\right) u^{2}+\rho u+\gamma
$$

for $f(u)=\frac{1}{8} c_{1}^{2} u^{3}+\frac{1}{2} c_{1}\left(c_{2}-b\right) u^{2}+2 \rho u+2 \gamma, g(u)=c_{1} u+c_{2}$, where $c_{1}, c_{2}, b, \rho$ and $\gamma$ are arbitrary constants with $c_{1} \neq 0$;

$$
\xi=-\frac{c_{1}}{2} u+b(t, x), \eta=-\frac{1}{4} c_{1}^{2} u^{3}+\frac{1}{2} c_{1} u^{2}\left(b(t, x)-c_{2}\right)+\rho(t, x) u+\gamma(t, x),
$$

for $f(u)=0, g(u)=c_{1} u+c_{2}$, where $b=b(t, x), \rho=\rho(t, x)$ and $\gamma=\gamma(t, x)$ are solutions to the system

$$
\begin{aligned}
& b_{t}-b_{x x}+\left(2 b-c_{2}\right) b_{x}-2 \rho_{x}=0, \\
& \rho_{t}-\rho_{x x}+c_{2} \rho_{x}+2 \rho b_{x}+c_{1} \gamma_{x}=0, \\
& \gamma_{t}-\gamma_{x x}+c_{2} \gamma_{x}+2 \gamma b_{x}=0,
\end{aligned}
$$

where $c_{1}$ and $c_{2}$ are arbitrary constants with $c_{1} \neq 0$.

Case 2: $r_{4}=0$.

In the case, the additional condition $r_{4}=\xi_{u}=0$ gives a first step simplification of the system $\mathcal{D}$ of determining equations. In order to further simplify the system, we use Theorem 2 to find more information on nontrivial nonclassical symmetry of the equation. For a $\Lambda=(\xi, \eta) \in Z(\mathcal{D})$, under transformation Equation (7), we have

$$
D^{\prime \prime}=\left\{\tau_{u}^{\prime}, \tau_{x}^{\prime}, \tau_{t}^{\prime}-2 \xi_{x} \tau^{\prime}\right\}
$$

It is clear that $\mathrm{Z}\left(\mathcal{D}^{\prime \prime}\right)=\varnothing$ if $\xi_{x x} \neq 0$. Hence, by Corollary 1 , we look for a nontrivial nonclassical symmetry of the form $\xi=a(x)$ with $a^{\prime \prime}(x) \neq 0$. Now, we suppose $\eta=b(t, x) u+c(t, x)$ from the $p_{1}=0$. These significantly simplify the system of determining equations.

For simplicity, denote $b=b(t, x), c=c(t, x)$ and $a=a(x)$.

Now, the rests of determining equations $p_{2}=0$ and $p_{4}=0$ become

$$
\begin{aligned}
& (u+A) f^{\prime}(u)+(2 B-1) f(u)+(E u+F) g(u)+H u=G \\
& (u+A) g^{\prime}(u)+B g(u)=K
\end{aligned}
$$

for $b \neq 0$, where

$$
\begin{aligned}
& A=c / b, B=a^{\prime} / b, K=\left(2 a a^{\prime}+2 b_{x}-a^{\prime \prime}\right) / b, H=\left(b_{x x}-b_{t}-2 b a^{\prime}\right) / b, \\
& E=-b_{x} / b, F=-c_{x} / b, G=\left(c_{t}-c_{x x}+2 c a^{\prime}\right) / b
\end{aligned}
$$


and

$$
\begin{aligned}
& f^{\prime}(u)+2 B_{1} f(u)+K_{1} g(u)=H_{1}, \\
& g^{\prime}+B_{1} g(u)=E_{1}
\end{aligned}
$$

for $b=0$ and $c \neq 0$, where

$$
B_{1}=a^{\prime} / c, K_{1}=-c_{x} / c, E_{1}=\left(2 a a^{\prime}-a^{\prime \prime}\right) / c, H_{1}=\left(2 c a^{\prime}+c_{t}-c_{x x}\right) / c .
$$

Since the $f$ and $g$ are functions of a single variable $u$, the coefficients $A, B, K, H, E, F, G, B_{1}, E_{1}, K_{1}$ and $H_{1}$ must be constants. After simple analysis, we can prove that Equation (19) does not admit a nontrivial nonclassical symmetry unless $g(u) \equiv 0, b=b(x) \neq 0$ and $c=c(x)$.

Under $g(u)=0$, Equations (24) become

$$
\begin{aligned}
& (u+A) f^{\prime}(u)+(2 B-1) f(u)+H u=G, \\
& K=2 a a^{\prime}+2 b^{\prime}-a^{\prime \prime}=0,
\end{aligned}
$$

with

$$
c=A b, a^{\prime}=B b, b^{\prime \prime}-2 a^{\prime} b=H b, 2 a^{\prime} c-c^{\prime \prime}=G b, A H+G=0 .
$$

Solving the first equation, we get

$$
f(u)=k(u+A)^{\mu}-\frac{H}{2 B}(u+A), \text { with } \mu=1-2 B \neq 0,
$$

and

$$
f(u)=k-H u \text {, with } 2 B-1=0,
$$

for arbitrary constant $k$.

It easily proves that the compatibility condition of Equation (27) for case Equation (28) of $f(u)$ is $B=-1$, i.e., $\mu=3$. Meantime, the $a=a(x)$ satisfies $3 a^{\prime \prime}-2 a a^{\prime}=0$ which further determines $b$ and $c$ from the equations in Equation (27). Consequently, we obtain nonclassical symmetries of Equation (19) as follows.

$$
\xi=a(x), \quad \eta=(u+A) b(x),
$$

with $b(x)=-a^{\prime}(x), c(x)=-A a^{\prime}(x)$ and

$$
\begin{aligned}
& a(x)=k_{1} \tanh \left(\left(x+3 c_{1}\right) k_{1} / 3\right), \text { for } f(u)=k(u+A)^{3}+H(u+A) / 2 \\
& a(x)=-3 /\left(x+3 c_{2}\right), \text { for } f(u)=k(u+A)^{3}
\end{aligned}
$$

where $c_{1}$ and $c_{2}$ are arbitrary constants and $k_{1}^{2}=-9 H / 4 \neq 0$.

In case Equation (29), the Equation (19) becomes a linear equation. The determining equations for nonclassical symmetries of the linear equation yield

$$
\xi=c(t, x), \quad \eta=a(t, x) u+b(t, x),
$$


where the functions $a=a(t, x), b=b(t, x)$ and $c=c(t, x)$ satisfy

$$
\begin{aligned}
& b_{t}-b_{x x}+2(b-k) c_{x}+k a+H b=0, \\
& a_{t}-a_{x x}+2(a+H) c_{x}=0, \\
& c_{t}-c_{x x}+2 c c_{x}+2 a_{x}=0 .
\end{aligned}
$$

It may be not possible to find the general solutions of the above PDE system. In the following, we give some special solutions.

Solving the system under the condition $b(t, x)=(-k / H) a(t, x)$, we obtain two groups of solutions:

$$
\begin{aligned}
& a(t, x)=\frac{2 c_{2}^{2}\left(c_{1}+2 c_{2} c_{4}\right) \tanh (T)+2 c_{2}\left(c_{4} c_{1}-2 c_{2} H\right)+c_{1}^{2}}{4 c_{2}^{2}}, \\
& c(t, x)=c_{4}-c_{2} \tanh (T) ;
\end{aligned}
$$

and

$$
\begin{aligned}
& a(t, x)=\frac{4 c_{2}^{4}-4 H c_{2}^{2}-c_{1}^{2}-6 c_{2}^{2}\left(2 c_{2}^{2} \tanh (T)^{2}+c_{1} \tanh (T)\right)}{4 c_{2}^{2}}, \\
& c(t, x)=-\frac{3 c_{2}^{2} \tanh (T)+c_{1}}{c_{2}}
\end{aligned}
$$

with $T=c_{1} t+c_{2} x+c_{3}$ in terms of arbitrary constants $c_{1}, c_{2} \neq 0$ and $c_{3}$.

In the special case where the functions $a=a(x), b=b(x)$ and $c=c(x)$ are independent of $t$, we have a additional group of solutions to the determining equations given by

$$
a(x)=\frac{c^{\prime}(x)-c^{2}(x)}{2}-H, b(x)=-\frac{k}{H} a(x), c(x)=\frac{3 x^{2}}{3 k_{1}-x^{3}},
$$

in terms of an arbitrary constants $k_{1}$.

Summarizing the above procedure, we obtain a nonclassical symmetry classification of Equation (19). We have proven that the equation admits nontrivial nonclassical symmetry when $f(u)$ is a polynomial with degree less than four in $u$ and $g$ is linear in $u$. The symmetries (30) with (31) and (32) with (35) were given in [11]. The symmetries (21), (22), (32) with (33) and (34) are new ones. The symmetries (23) are more general than those given in [11].

Moreover, here in the case $g=0$, we recovered the symmetry classification results in [11] obtained by P. A. Clarkson and E. L. Mansfield who directly treat the system of determining equations by the Gröebner basis method. In the case $g(u)=\lambda u$, we recovered the results in [22] given by Roman Cherniha who dealt with the determining system by direct calculation. Here, we solve the problem for Equation (19) by an alternative method using Theorems 2 and 3 and Corollaries 1 and 2 in general sense for arbitrary $f(u)$ and $g(u)$ and show that only in the case when $g$ is linear and $f(u)$ is cubic polynomials in $u$, the class of Equations (19) admits a nontrivial nonclassical symmetry. In particular, compared with the ways for symmetry classification problem existing in literatures [10-12,22], our method avoids a lot calculations for dealing with the determining equations.

Of physical interest is that there are many important equations, such as the Huxley equation, the Chaffee-Infante or Newell-Whitehead equation, the generalized Fisher's equations with $k=3$ or $p=2$, the generalized KPP equation, the Fitzhugh-Nagumo equation, Burgers equation, the Burgers-Huxley equation, that admit nontrivial nonclassical symmetries. Nevertheless, equations such as the generalized Burgers-Fisher equation, the generalized Burgers-Huxley equation $(c \neq 1)$, the Fisher's equation, the generalized Fisher's equations with $k \neq 3$ and $p \neq 2$ do not admit nontrivial nonclassical symmetries. 


\subsection{Nonclassical Symmetry of a KdV-Type Equations}

Consider a KdV-type equations in soliton theory [6,12] given by

$$
u_{t}+3 v v_{x}=0, v_{t}+2 v_{x x x}+2 u v_{x}+u_{x} v=0
$$

The system of determining polynomials for the classical symmetry $\mathcal{X}^{\prime}=\tau^{\prime} \partial_{t}+\xi^{\prime} \partial_{x}+\eta^{\prime} \partial_{u}+\phi^{\prime} \partial_{v}$ of the equations is given by

$$
\mathcal{D}^{\prime}=\left\{\begin{array}{l}
\tau_{v}^{\prime}, \tau_{u}^{\prime}, \tau_{x}^{\prime}, 3 \eta^{\prime}+2 u \tau_{t}^{\prime}, \\
\eta_{v}^{\prime}, \eta_{x}^{\prime}, \eta_{t}^{\prime}, \eta^{\prime}-u \eta_{u}^{\prime}, u \phi^{\prime}-v \eta^{\prime}, \\
\xi_{v}^{\prime}, \xi_{u}^{\prime}, \xi_{t}^{\prime}, \eta^{\prime}+2 u \xi_{x}^{\prime} .
\end{array}\right.
$$

and it has $Z\left(\mathcal{D}^{\prime}\right)=\left\{\tau^{\prime}, \xi^{\prime}, \eta^{\prime}, \phi^{\prime}\right\}$, where

$$
\tau^{\prime}=c_{2}-3 c_{1} t, \xi^{\prime}=c_{3}-c_{1} x, \eta^{\prime}=2 c_{1} u, \phi^{\prime}=2 c_{1} v,
$$

and $c_{1}, c_{2}, c_{3} \in \mathbb{R}$ are arbitrary constants. It yields classical symmetries

$$
\mathcal{X}_{1}^{\prime}=-\frac{1}{2}\left(3 t \partial_{t}+x \partial_{x}\right)+u \partial_{u}+v \partial_{v}, X_{2}^{\prime}=\partial_{t}, \mathcal{X}_{3}^{\prime}=\partial_{x}
$$

of Equations (36). By our given algorithm, we have the set $C=\left\{r_{1}, \cdots, r_{9}\right\}$, where

$$
\begin{array}{ll}
r_{1}=3 \eta \xi-2 u \xi_{t}, & r_{4}=3 \eta^{2}-2 u \eta_{t}, \\
r_{2}=2 u \xi_{x}+\eta, & r_{5}=\eta-u \eta_{u}, \\
r_{3}=u \phi-v \eta, & r_{6}=\xi_{u}, r_{7}=\xi_{v}, r_{8}=\eta_{x}, r_{9}=\eta_{v} .
\end{array}
$$

The system $\mathcal{D}$ of determining polynomials of the nonclassical symmetry of the equations is consists of 11 strongly nonlinear equations, variable coefficients and long expressions for differential polynomials. We can establish the identities Equation (10) between the system $\mathcal{D}$ and $C$ by our given algorithm. Due to complexity and massive scale, we here omit the expressions of the connections. The system $\mathcal{D}$ is difficult to deal with directly. Nevertheless, we can get some specific nonclassical symmetries of Equation (36) by using Corollaries 1 and 2.

We notice that there are three identities in Equation (10) that only involve the $r_{6}$ and $r_{7}$. In order to reduce the system, we deliberately set $r_{6}=r_{7}=0$. In addition, we take some analysis on nonclassical symmetry of the equations. From the set $\mathcal{D}^{\prime \prime}=\left\{\tau_{v}^{\prime}, \tau_{u}^{\prime}, \tau_{x}^{\prime}, 3 \eta^{\prime}+2 u \tau_{t}^{\prime}\right\} \subset C^{\prime}$, we see that $Z\left(D^{\prime \prime}\right)=\varnothing$ if one of $\eta_{t}, \eta_{x}, \eta_{u u}$ and $\eta_{v}$ is not zero. Hence they may provide sufficient conditions for existence of nonclassical symmetries of Equations (36) by Theorem 2. With considering the sufficient conditions and for further simplifying the determining equations, we take $D_{u} r_{2}=2 u \xi_{x}+\eta_{u}, D_{v} r_{2}=\eta_{v}, D_{u} r_{5}=-u \eta_{u u}$ and $D_{v v} r_{3}=\phi_{v v}$ as more conditions by Corollary 2. Under these conditions the infinitesimal functions are simplified as

$$
\xi=B(t, x), \eta=-2 B_{x}(t, x) u+B(t, x), \phi=G(t, x) v+H(t, x) .
$$

With symbolic computations, substituting the solutions into the system of determining equations $\mathcal{D}=0$ and setting the coefficients of $u$ and $v$ as zero, we obtain solutions

$$
\begin{aligned}
& \xi=\frac{1}{3} x(\operatorname{ct}(t)+1) c_{1}+e^{c_{1} t} \operatorname{cs}(t) c_{3}, \\
& \eta=\left(\frac{1}{3} x(\operatorname{ct}(t)+1) c_{1}+e^{c_{1} t} \operatorname{cs}(t) c_{3}-\frac{2}{3} u(\operatorname{ct}(t)+1)\right) c_{1} \\
& \phi=\frac{1}{3} v(1-2 \operatorname{ct}(t)) c_{1} \\
& \xi=c_{2} e^{2 c_{1} t} \\
& \eta=c_{1} c_{2} e^{2 c_{1} t} \\
& \phi=c_{1} v
\end{aligned}
$$


in which $(\operatorname{ct}(\mathrm{t}), \operatorname{cs}(\mathrm{t}))=\left(\tanh \left(c_{1} t-c_{2}\right), \operatorname{sech}\left(c_{1} t-c_{2}\right)\right)$ and $(\operatorname{ct}(\mathrm{t}), \operatorname{cs}(\mathrm{t}))=\left(\operatorname{coth}\left(c_{1} t-c_{2}\right), \operatorname{csch}\left(c_{1} t-c_{2}\right)\right)$, and $c_{1} \neq 0, c_{2}, c_{3}$ are arbitrary constants. Obviously, these solutions are not in $\mathrm{Z}\left(\mathcal{D}^{\prime}\right)$ since $\eta_{t} \neq 0$.

These recovered the results in [12]. Compared with the calculation of the method in [12], the calculation here is simpler.

\section{Conclusions}

Some relationships (Theorems 2-4 and Corollaries 1 and 2) between the classical and nonclassical symmetries of a PDE are given. An important innovation idea presented in the connections is that additional information on the nonclassical symmetry to be determined is obtained directly from the inconsistence of the determining system of classical symmetry of the PDE. Another more interesting point is the use of the equivalent transformation Equation (7) and deriving the set $C$. The set $C$ connects such symmetries of the PDE in terms of their determining polynomials. In addition, Wu's reduction formula and algorithm play fundamental rules in deriving these results. In particular, an algorithm based on the reduction algorithm is given for constructing connections Equation (10).

These obtained connections efficiently yield sufficient conditions on the existence of the nonclassical symmetries of a PDEs, which make it easier to solve the system of nonlinear determining equations. Because these connections provide further information for the nonclassical symmetry, it is not necessary to calculate the characteristic set of the system of the determining equations at the beginning. Hence the computation is much more cheaper. Consequently, the connections give an alternative way to obtain nontrivial nonclassical symmetry of a PDE and improve the efficiency of the algorithms given in literatures. Moreover, the connections yield a way to better understand the relationship between classical and nonclassical symmetries of given PDEs. The higher efficiency applications of the obtained results for determining nontrivial nonclassical symmetries yielded new nonclassical symmetries for a class of the generalized Burgers equations.

Author Contributions: Investigation, G.B.; Methodology, C.T. and L.T. All authors have read and agreed to the published version of the manuscript.

Funding: This research was funded by Natural science foundation of China, under grand numbers: 11571008 .

Acknowledgments: The first two authors are supported by Natural science foundation of China, under grand numbers: 11571008. George Bluman acknowledges financial support from the National Sciences and Engineering Research Council of Canada. Authors thanks reviewers' kind and valuable comments and suggestions.

Conflicts of Interest: The authors declare no conflict of interest.

\section{References}

1. Bluman, G.W.; Cole, J.D. The general similarity solution of the heat equation. J. Math. Mech. 1969, 18, 1025-1042.

2. Bluman, G.W.; Kumei, S. Symmetries and Differential Equations; Springer: New York, NY, USA, 1989.

3. Olver, P.J. Applications of Lie Groups to Differential Equations, 2nd ed.; Springer: New York, NY, USA, 1993.

4. Clarkson, P.A. Nonclassical symmetry reductions of the Boussinesq equation. Chaos Solitons Fractals 1995, 12, 2261-2301. [CrossRef]

5. Nucci, M.C.; Clarkson, P.A. The nonclassical method is more general than the direct method for symmetry reductions. An example of the Fitzhugh-Nagumo equation. Phys. Lett. A 1992, 164, 49-56. [CrossRef]

6. Baumann, G.; Haager, G.; Nonnenmacher, T.F. Applications of nonclassical symmetries. J. Phys. A: Math. Gen. 1994, 27, 6479-6493. [CrossRef]

7. Mansfield, E.L. Applications of the differential algebra package diffgrob2 to classical symmetries of differential equations. J. Symb. Comput. 1993, 5-6, 517-533.

8. Clarkson, P.A.; Mansfield, E.L. Open problems in symmetry analysis. Contemp. Math. 2001, 285, $195-205$.

9. Ibragimov, N.H. CRC Handbook of Lie Group Analysis of Differential Equations. Vol 2: Applications in Engineering and Physical Sciences; CRC Press: London, UK; Tokyo, Janpan, 1994.

10. Clarkson, P.A.; Mansfield, E.L. Algorithms for the non-classical method of symmetry reductions. SIAM J. Appl. Math. 1994, 54, 1693-1719. [CrossRef] 
11. Clarkson, P.A.; Mansfield, E.L. Symmetry reductions and exact solutions of a class of nonlinear heat equations. Phys. D Nonlinear Phenom. 1994, 70, 250-288. [CrossRef]

12. Temuer, C.; Bluman, G. An algorithmic method for showing existence of nontrivial non-classical symmetries of partial differential equations without solving determining equations. J. Math. Anal. Appl. 2014, 411, 281-296.

13. Temuer, C. New algorithm for classical and nonclassical symmetry of a PDE based on Wu's method. Sci. Sin. Math. 2010, 40, 331-348. (In Chinese)

14. Temuer, C.; Pang J. An algorithm for the complete symmetry classification of differential equations based on Wu's method. J. Eng. Math. 2010, 66, 181-199.

15. Bai, T.; Temuer, C. A Potential Constraints Method of Finding Nonclassical Symmetry of PDEs Based on Wu's Method. Adv. Math. Phys. 2019, 2019, 272347. [CrossRef]

16. Ritt, J.F. Differential Algebra; American Mathematical Society: Providence, RI, USA, 1950; Volume 33.

17. Kolchin, E.R. Differential Algebra and Algebraic Groups; Academic Press: New York, NY, USA; London, UK, 1973.

18. Wu, W.T. On the foundation of algebraic differential geometry. Syst. Sci. Math. Sci. 1989, 2, $289-312$.

19. Wu, W.T. Mathematics Mechanization; Mathematics and Its Applications; Science Press: Beijing, China; Kluwer Academic Publishers: Dordrecht, The Netherlands; Boston, MA, USA; London, UK, 2000; Volume 489.

20. Gao, X.S.; Wang, D.K.; Liao, Q.; Yang, H. Equation Solving and Machine Proving-Problem Solving with MMP; Science Press: Beijing, China, 2006. (In Chinese)

21. Temuer, C. An algorithmic theory of reduction of a differential polynomial system. Adv. Math. 2003, 32, $208-220$.

22. Cherniha, R. New Q-conditional symmetries and exact solutions of some reaction-diffusion-convection equations arising in mathematical biology. J. Math. Anal. Appl. 2007, 326, 783-799. [CrossRef]

23. Serov, M.I.; Cherniha, R.M. Lie symmetries and exact solutions of nonlinear equations of heat conductivity with convection term. Ukr. Math. J. 1997, 49, 1423-1443. [CrossRef]

24. Cherniha, R.; Serov, M. Symmetries, ansatze and exact solutions of nonlinear second-order evolution equations with convection terms, II. Eur. J. Appl. Math. 2006, 17, 597-605 [CrossRef]

(C) 2020 by the authors. Licensee MDPI, Basel, Switzerland. This article is an open access article distributed under the terms and conditions of the Creative Commons Attribution (CC BY) license (http:/ / creativecommons.org/licenses/by/4.0/). 